\title{
J.B.Watson, a psicologia experimental e o condutismo 100 anos depois
}

\section{J.W.Watson, experimental psychology and behaviorism 100 years later}

\author{
Rubén Ardila* \\ Universidad Nacional de Colombia, Bogotá, Colômbia.
}

\begin{abstract}
RESUMO
Apresenta-se neste artigo uma análise do trabalho publicado por Watson em 1913, denominado "Manifesto Behaviorista", que procurou ser o fundamento de uma psicologia baseada nas ciências naturais, na experimentação e no estudo objetivo do comportamento. Indicam-se suas origens, o contexto no qual se desenvolveu seu autor e a forma como o condutismo evoluiu e se diversificou. Destaca-se o legado de Watson, as críticas recebidas e sua avaliação histórica.
\end{abstract}

Palavras-chave: Condutismo, Psicologia experimental, Watson.

\begin{abstract}
An analysis is presented of the paper "Psychology as the behaviorist views it" published by Watson in 1913 that has been called "The behaviorist Manifest", that wanted to be the foundation of a psychology based on natural sciences, experimentation and the objective study of behavior. The origin, context, the autor of the work and the way in which behaviorism developed and diversified, are indicated. Watson's legacy is presented, including criticisms and historical evaluation.
\end{abstract}

Key words: Behaviorism, Experimental psychology, Watson

\section{I ntrodução}

No ano de 2013 comemoram-se 100 anos da publicação do artigo de John B. Watson (1878-1958), intitulado "Psychology as the behaviorist views it" (1913). Foi este polêmico trabalho, de grande influência na comunidade científica, que deu origem a uma das chamadas "escolas psicológicas", o condutismo". Um século depois, continua sendo considerada uma das publicações mais influentes em toda a história da psicologia. Em vários países, incluindo nações europeias, latino-americanas, os Estados Unidos, o Japão, etc., essa efeméride teve muita repercussão e gozou de grande importância.

Watson nasceu em Greenville, Carolina do Sul, estudou na Furman University e mais tarde na Universidade de Chicago. Seu interesse 
pela filosofia, e especificamente pela epistemologia, conduziu-o à psicologia experimental. Foi muito influenciado pelas ideias de John Dewey, James Angell e os funcionalistas da Escola de Chicago. Estudou sob a direção de Angell e de um neurologista, Henry Donaldson, que tinha desenvolvido a linhagem de ratos Wistar. Depois de doutorar-se em 1903, com uma tese intitulada "Animal education: the psychical development of the white rat" permaneceu um tempo em Chicago e depois transferiu-se (1908) à Universidade John Hopkins, em Baltimore. Ali realizou a maior parte de suas pesquisas experimentais, tanto com animais quanto com participantes humanos; escreveu artigos científicos e livros; polemizou com os psicólogos tradicionais introspeccionistas que consideravam a psicologia a ciência da mente e não da conduta; foi presidente da American Psychological Association (APA) em 1915; e saiu da Universidade em 1920 por um grande escândalo que mudou em parte suas perspectivas vitais. Além de trabalhos experimentais, realizou também observações etológicas e foi considerado o "protoetólogo".

Daquele momento em diante dedicou-se à psicologia aplicada, escreveu sobre educação infantil, publicidade e propaganda, aspectos populares da psicologia, foi um pioneiro muito reconhecido em campos aplicados e um promotor das possibilidades da psicologia no novo século. Continuou atualizando seus livros, sendo editor de revistas científicas e membro de comitês editoriais. Não é totalmente verdade que ele teve que abandonar o mundo acadêmico, mas sim que o foco de seu trabalho passou a ser a psicologia aplicada e não a pesquisa básica.

\section{0 "manifesto behaviorista"}

No momento de publicar seu trabalho, que se denominou "O Manifesto Behaviorista" (ou Manifesto Condutista), Watson tinha 35 anos e trabalhava na Universidade John Hopkins. Era uma figura respeitada da psicologia norte-americana e tinha sido reconhecido internacionalmente como um dos líderes nesse campo. Seu trabalho fora muito influenciado pela filosofia naturalista, por Darwin (ver BOAKES, 1984) e a evolução, considerando a psicologia como ciência natural e também como uma disciplina com importantes aplicações sociais, no mundo da educação infantil, na família, no trabalho, na publicidade e em outros temas similares.

A psicologia "objetiva" não começa com Watson, já que encontramos antecedentes em Sechenov, Pavlov e Bechterev. Nos Estados Unidos, Thorndike e os psicólogos comparativos tinham realizado importantes trabalhos dentro da perspectiva objetiva, e não mentalista, da psicologia. O mesmo acontecera na Inglaterra. Na Argentina, José 
Ingenieros também se dedicou a estudar o comportamento, e o mesmo tinha feito Piéron na França. Mas Watson teve grande impacto na comunidade científica internacional, e representou um marco no estudo do comportamento, ou da psicologia comportamental, como foi dito algumas vezes.

Seu artigo de 1913, Psychology as the behaviorist views it (WATSON, 1913) representou uma crítica à psicologia mentalista, bem como uma proposta a partir de uma nova perspectiva, centrada no estudo objetivo da conduta, sem levar em conta a consciência nem utilizar a introspecção como método para encontrar dados válidos em psicologia. Para Watson, a psicologia não precisava da introspecção nem da consciência, da alma ou da mente, do mesmo modo como a física ou a química não necessitam delas. Poderíamos dizer que ele propôs uma "psicologia em terceira pessoa", enquanto a de Wundt tinha sido em primeira pessoa (o participante fazendo introspecção sobre o conteúdo de sua consciência), e a psicologia de Freud em segunda pessoa (o terapeuta analisando as associações do paciente por meio da transferência). A psicologia de Watson em terceira pessoa se constituía como uma ciência natural, de laboratório, sem pressupostos filosóficos especulativos.

As críticas formuladas por Watson contra a psicologia introspeccionista afirmavam que, para que a psicologia pudesse transformar-se em uma ciência natural, seria preciso que abandonasse o estudo da consciência e o método introspectivo. A conduta dos seres humanos e dos animais não humanos podia ser estudada sem referências à consciência. Em sua proposta de reconceitualizar a psicologia e dar a ela um novo impulso, Watson (1913) afirmou que a psicologia vista por um condutista é:

[...] um ramo puramente objetivo e experimental da ciência natural. Seu objetivo teórico é a previsão e o controle da conduta. A introspecção não faz parte essencial de seus métodos [...]. O condutista [...] não reconhece uma linha divisória entre o homem e o animal. O comportamento do homem, com todo seu refinamento e complexidade, forma somente uma parte do esquema total de pesquisa do condutista (p. 158).

Uma das características centrais do Manifesto Behaviorista foi sua ênfase no ambiente. Embora Watson tenha se referido em muitas ocasiões a fatores biológicos, e especialmente neurofisiológicos, em seus trabalhos com animais, crianças e adultos, a ênfase se centrou no papel do ambiente. Ele nunca descartou o papel da biologia na conduta. No que tange ao ambiente, deu-lhe uma importância primordial. Sua conhecida frase sobre esse assunto diz o seguinte: 
Deem-me uma dúzia de crianças saudáveis e bem formadas e meu mundo específico para criá-las, e eu me comprometo a escolher uma delas ao acaso e treiná-la para que chegue a ser qualquer tipo de especialista que escolher: médico, advogado, artista, comerciante, e inclusive mendigo ou ladrão, sem levar nem um pouco em conta seus talentos, capacidades, tendências, habilidades, vocação ou a raça de seus antepassados (WATSON, 1930, p. 104).

Esse ambientalismo extremo implicava uma filosofia otimista em relação com o comportamento humano e a sociedade. Os seres humanos podem ser modificados - no sentido adaptativo ou não adaptativo - e não estão à mercê de contingências biológicas (ou genéticas, em termos contemporâneos). Somos aperfeiçoáveis e modificáveis, não há um determinismo genético e "a biologia não é um destino" (como foi dito em épocas recentes). Se decidirmos fazêlo, podemos ter um ser humano cada vez melhor e uma sociedade também cada vez melhor.

\section{Evolução do condutismo}

A proposta de Watson foi recebida com grande interesse por muitos psicólogos e outros cientistas, e com uma completa rejeição por parte de outros. Tal fato serve para polarizar os partidários e os inimigos do condutismo, e dar forma a um "paradigma". O condutismo se contrapôs ao estruturalismo, ao funcionalismo, à psicanálise e a outras maneiras de conceitualizar os fenômenos psicológicos. Transformou-se em uma "escola" psicológica, que com o passar do tempo evoluiu, transformou-se em um "sistema" e se diluiu parcialmente, dentro da psicologia e também fora dela, como um campo específico e diferente do conhecimento (ver ARDILA, 1965, 2006a, 2006b, 2010a, 2010b).

A recepção do condutismo, e especificamente do artigo de 1913, na Psychological Review foi analisada detalhadamente por Samelson (1981), que concluiu que não foi tão positiva como se supunha. Psicólogos destacados por M. W. Calkins e E. Thorndike fizeram muitas críticas à nova proposta.

Mais tarde foram sugeridos condutismos alternativos, associados aos nomes de Hull, Tolman, Skinner, Kantor e outros pesquisadores e teóricos, e, mais recentemente, aos nomes de Rachlin, Baum, Hayes, Ribes, Staats, entre outros (ver PÉREZ-ACOSTA; GUERRERO; LÓPEZ, 2002). Em muitos casos, o modelo estímulo-resposta (E-R) descartou-se totalmente e foi substituído pelo modelo estímuloresposta-consequência. Em outros casos, se introduziram variáveis organísmicas (E-O-R), internas, psicológicas, etc. Mas se manteve a ênfase no estudo científico do comportamento. 


\section{Condutismo e cultura}

No início do século XX, nos Estados Unidos, ocorriam várias mudanças culturais, e se desenvolvia uma mentalidade baseada na autonomia, no individualismo, na necessidade de controlar o ambiente e de construir uma sociedade com base nos princípios da ciência e da tecnologia da época, e de planejar o futuro. Uma sociedade cada vez mais urbana requeria novos modos de manter a ordem e o controle social. A psicologia era uma nova disciplina que estava começando a tomar forma e dar origem a aplicações profissionais - modestas no começo - nos campos da educação, do estudo da criança, da indústria, da publicidade e no mundo do trabalho. A avaliação e a medição, os testes psicológicos e seu papel na sociedade, começavam a ser reconhecidos (ver CAPSHEW, 1999 e HERMAN, 1995 para análises históricas sobre o papel da psicologia como ciência e como profissão na formação da cultura).

O condutismo de Watson, com sua ênfase na ciência natural e com suas aplicações potenciais aos mais diversos campos da atividade humana, encaixou muito bem na cultura norte-americana da segunda década do século $X X$. O movimento iniciado por Watson encontrou espaço no contexto da história social e cultural dos Estados Unidos no momento, como assinalou detalhadamente Buckley (1989) em sua biografia de Watson. A nova psicologia, com ênfase no prognóstico e no controle do comportamento, dava uma resposta à sociedade da época. O "evangelho" do sucesso por meio do autocontrole, e a preocupação nacional com a eficiência e a ordem durante a transição de uma sociedade individualista a uma sociedade corporativa, estavam perfeitamente de acordo com as propostas originais de Watson (ver também COHEN, 1979).

Com o passar do tempo, essas ideias se estenderam a outros contextos culturais e se internacionalizaram. Sem dúvida a modernidade, a valorização da ciência natural, o grande desenvolvimento da psicologia a nível internacional e sua busca de "credibilidade" no mundo acadêmico e no mundo aplicado, fizeram com que o condutismo fosse reconhecido em outras culturas diferentes da norte-americana. Possivelmente as afirmações exageradas de Watson, seu otimismo exuberante e seu "mecanicismo", não foram bem recebidos em todos os lugares, e pelo contrário, as ideias de Skinner e da análise experimental do comportamento tiveram muito mais influência. A relação entre o condutismo clássico de Watson e o condutismo radical de Skinner foi muito estudada. O condutismo que chegou mais longe foi o de Skinner, como assinalamos anteriormente (ARDILA, 2006a), mas é 
claro que este deve muito à conceitualização original de Watson, embora se diferenciasse dela em vários aspectos fundamentais.

\section{O legado de Watson}

No obituário publicado por Woodworth (1959), este afirma que os principais temas estudados experimentalmente por Watson foram: o desenvolvimento comportamental em relação com o desenvolvimento neurológico no rato branco; os sinais sensoriais utilizados ao aprender a mover-se em um labirinto; o papel do sentido sinestésico no controle do comportamento; a visão das cores em várias espécies de animais; os "instintos" das andorinhas-do-mar; e o desenvolvimento emocional precoce da criança.

Nesse último ponto, Watson realizou vários trabalhos sobre a origem das emoções, afirmando que três delas são básicas: o medo, a raiva e o amor, das quais derivam as outras, em estágios posteriores de desenvolvimento. Embora no estudo experimental das emoções tenha se avançado muito desde a época de Watson, sem dúvida seus estudos ainda são parcialmente válidos na atualidade.

Podemos acrescentar à descrição de Woodworth sobre as contribuições de Watson relacionadas com a psicologia aplicada, as pautas de educação das crianças, a educação em geral, a publicidade, a eficiência, a modificação do comportamento e outros temas afins. Podem ser mencionados ainda seus estudos sobre linguagem e pensamento.

Entretanto, o mais importante é sua conceitualização a respeito da psicologia como ciência do comportamento, como um campo de pesquisa de problemas objetivos que estão no mundo real, e sua luta contra as especulações de qualquer índole. Provavelmente o principal livro publicado a respeito de Watson e sua obra é Modern Perspectives on John B. Watson and Classical Behaviorism (TODD; MORRIS, 1994). E quanto ao condutismo no momento atual, o livro de García Cadena (2007), Introducción al Conductismo Contemporáneo.

O que ficou hoje de Watson e do Manifesto Behaviorista, 100 anos depois? Mantem-se a sua ênfase na psicologia como ciência natural (além de ciência social). Também sua definição da psicologia como ciência do comportamento, que é a mais utilizada em nível mundial. Permanece sua insistência na continuidade psicológica entre a espécie humana e outras espécies, sua crença nas aplicações atuais e potenciais, e suas contribuições para a tarefa de conseguirmos uma sociedade melhor. Pelo contrário, o estudo da consciência retornou ao "mainstream" da psicologia científica, embora não tenha ocorrido o mesmo com a introspecção como método primitivo para obter dados válidos. As ideias de Watson sobre a educação das crianças não 
sobreviveram às pesquisas posteriores e não têm muita validade. Seus estudos sobre as emoções, suas observações etológicas, as bases da modificação do comportamento que ele e sua esposa Rosalie Rayner propuseram, continuam fazendo parte do acervo terapêutico dos psicólogos clínicos do século XXI.

Watson foi um produto de sua época e do Zeitgeist, como somos todos os seres humanos. Mas também foi um homem que mudou o momento em que viveu, como muito poucos fazem. Um homem que lutou contra a corrente, teve uma vida cheia de altos e baixos, problemas, sucessos; uma vida intensa e variada. Sua avaliação histórica pode resumir-se nas palavras de Gustav Bergmann (1956):

\begin{abstract}
Somente inferior a Freud [...] Watson é a figura mais importante na história do pensamento psicológico durante a primeira metade do presente século [...]. Entre os psicólogos, a sólida base que foi a contribuição de Watson aceitou-se amplamente; seus erros foram esquecidos [...]. Watson não é somente um psicólogo experimental [...], mas também um pensador sistemático [...] um metodólogo. Foi nessa área que realizou sua contribuição mais importante (p.275).
\end{abstract}

Sendo assim, é um fato que sua obra deve considerar-se dentro de um contexto histórico, em uma linha de pensamento ampla, que requer avaliar criticamente todas as suas contribuições e separar o que continua válido do que foi um produto da época e do entusiasmo de seu criador, e não sobreviveu ao passar do tempo.

\title{
Referências
}

ARDILA, R. Behaviorismo: hacia una psicología científica. Revista de Psicología, Bogotá, v. 10, n. 2, p. 85-91, 1965.

Behavior analysis in an international context. In: BROCK, A.

C. (Ed.), I nternationalizing the history of psychology. New York: New York University Press, 2006a, p. 112-132.

The experimental synthesis of behaviour. International

Journal of Psychology, n. 41, p. 462-467, 2006b.

. Psychology and behavior analysis. The nature of the controversy. In: SCHWARZER, R.; FRENSCH, P. A. (Eds.). Personality, human development and culture. International perspectives in psychological science. Vol. 2. Hove, UK: Psychology Press, 2010a, p. 263-274.

- La unidad de la psicología. El paradigma de la síntesis experimental del comportamiento. Revista Mexicana de Investigación en Psicología, v. 2, n. 2, p. 72-83, 2010 b.

BERGMANN, G. The contribution of John B. Watson. Psychological Review, n. 63, p. 265-276, 1956. 
BOAKES, R. A. From Darwin to behaviorism. Cambridge: Cambridge University Press, 1984.

BUCKLEY, K. W. Mechanical man. John Broadus Watson and the beginning of behaviorism. New York: Guilford Press, 1989.

CAPSHEW, J. H. Psychologists on the march. Cambridge: Cambridge University Press, 1999.

COHEN, D. J. B. Watson, the founder of behaviourism. A biography. London: Routhledge \& Kegan Paul, 1979

GARCÍA CADENA, C. H. Introducción al conductismo contemporáneo. México, D. F.: Editorial Trillas, 2007.

HERMAN, E. The romance of American psychology. Political culture in the age of experts. Berkeley: University of California Press, 1995.

PÉREZ-ACOSTA, A.; GUERRERO, F.; LÓPEZ LÓPEZ, W. Siete conductismos contemporáneos: una síntesis verbal y gráfica. International Journal of Psychology and Psychological Therapy, n. 2, p. 103-113, 2002.

SAMELSON, F. Struggle for scientific authority: The reception of Watson's behaviorism, 1913-1920. Journal of the History of the Behavioral Sciences, n. 17, p. 399-425.

TODD, J. T.; MORRIS, E. K. (Eds.), Modern perspectives on J ohn B. Watson and classical behaviorism. Westport, Conn.: Greenwood Press, 1994.

WATSON, J. B. Psychology as the behaviorist views it. Psychological Review, n. 20, p. 158-177, 1913.

. Psychology from the standpoint of a behaviorist. Philadelpia: Lippincott, 1919 [1924].

. Behaviorism. Ed. rev. New York: Norton, 1930.

WOODWORTH, R. S. John B. Watson. American Journal of Psychology, n. 72, p. 301-310, 1959.

Endereço para correspondência

Rubén Ardila

Carrera 16 No. $94-$ A- 23

Bogotá, D.C. 220221

Colombia

Endereço eletrônico: ruben.ardila@etb.net.co, psycholo@etb.net.co

Recebido em: 28/06/2013

Aceito para publicação em: 05/08/2013

Acompanhamento do processo editorial: Ana Maria Jacó Vilela

\section{Notas}

* Psicólogo en la Universidad Nacional de Colombia y el Ph.D. en Psicología Experimental en la University of Nebraska-Lincoln (USA).

${ }^{1}$ Em português, a nomenclatura utilizada para referir-se aos estudos de Watson é variável: behaviorismo, comportamentalismo ou condutismo.(N.T.) 